\title{
Interactions entre le type de plants et la sylviculture dans la restauration de sapinières sous forte pression de broutement
}

\author{
Par Maxime Brousseau 1,2, Jean-Pierre Tremblay 1,2,3, Vincent Roy ${ }^{4}$ et Nelson Thiffault 1,2,4*
}

\begin{abstract}
RÉSUMÉ
Les herbivores transforment les écosystèmes, particulièrement suite aux introductions en milieux insulaires. La plantation de sapins baumiers (Abies balsamea) est réalisée sur l'île d'Anticosti (Québec, Canada) pour restaurer ou maintenir des composantes essentielles de l'habitat du cerf de Virginie (Odocoileus virginianus), une espèce faunique qui supporte une forte activité économique. Dans ce contexte, notre objectif était d'évaluer les effets simples et combinés du dégagement, du type de plants et du cerf sur la survie et les dimensions du sapin baumier. Nous avons évalué ces effets pendant 8 ans dans un dispositif expérimental en blocs complets. Nos résultats indiquent qu'à lîle d'Anticosti, il n'est pas utile de procéder à des dégagements mécaniques; le broutement sur les compétiteurs a eu un effet similaire à celui du dégagement pour les plants. Nos observations supportent l'utilisation de plants de moyennes dimensions, lesquels ont atteint une hauteur à laquelle les flèches terminales étaient hors de la portée du cerf, tout en réduisant les coûts de production par rapport aux plants de fortes dimensions. Finalement, la chasse sportive en enclos de gestion a été efficace pour réduire le broutement sur les sapins; nous n’avons détecté aucun effet des cerfs sur leur survie et leur taille.
\end{abstract}

Mots clés : cerf de Virginie, broutement, dégagement mécanique, compétition, sylviculture, type de plants, plantation

\begin{abstract}
Large herbivores transform ecosystems, especially following introduction into island environments. Plantations of balsam fir (Abies balsamea) were established on Anticosti Island (Quebec, Canada) to restore or maintain essential components of white-tailed deer (Odocoileus virginianus) habitat, a species that supports strong economic activities. In this context, our objective was to evaluate the simple and combined effects of release, stock type, and deer on balsam fir seedling survival and size. We evaluated these effects over eight years using a complete block experimental design. Our results show that on Anticosti Island, mechanical release is not useful; browsing on competing species had a similar effect to release on the planted seedlings. Our observations support the use of medium-size planting stock which reached heights sufficient to protect terminal shoots from browsing, while reducing production costs relative to large stock seedlings. Finally, sport hunting in the management enclosure efficiently reduced browsing on planted seedlings; we did not detect any effects of white-tailed deer on seedling survival and size.
\end{abstract}

Key words: white-tailed deer, browsing, mechanical release, competition, silviculture, stock type, plantation

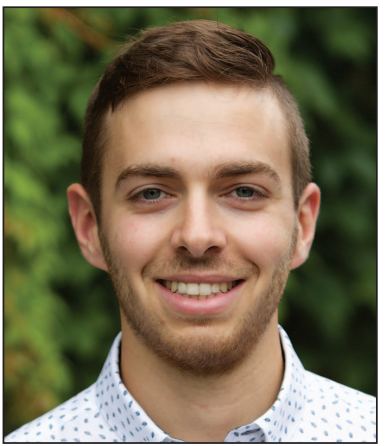

Maxime Brousseau

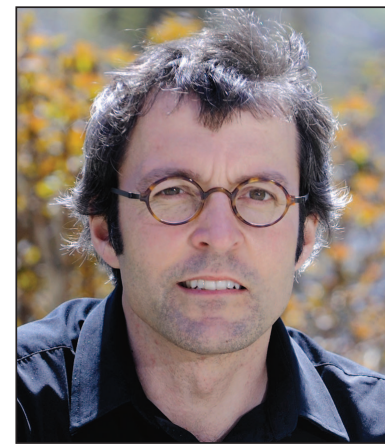

Jean-Pierre Tremblay

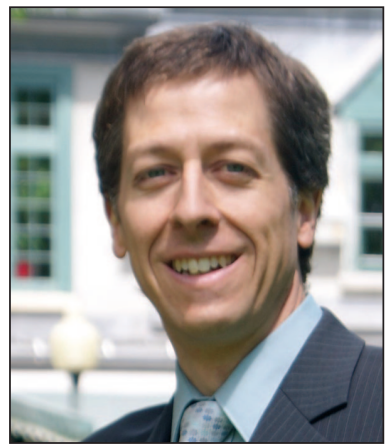

Vincent Roy

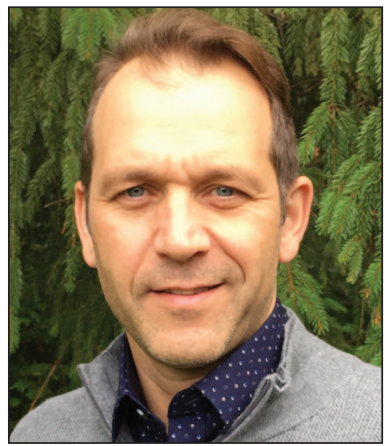

Nelson Thiffault

\footnotetext{
${ }^{1}$ Chaire de recherche industrielle CRSNG en aménagement intégré des ressources de l'île d'Anticosti et Département de biologie, 1045 avenue de la Médecine, Université Laval, Québec, QC G1V 0A6, Canada

${ }^{2}$ Centre d'étude de la forêt, Université Laval, Québec, QC G1V 0A6, Canada

${ }^{3}$ Centre d'études Nordiques Université Laval, Québec, QC G1V 0A6, Canada

${ }^{4}$ Centre canadien sur la fibre de bois, Service canadien des forêts, Ressources naturelles Canada, 1055, rue du P.E.P.S., C.P. 10380, succ. Sainte-Foy, Québec, QC G1V 4C7, Canada

*Auteur de correspondance : nelson.thiffault@canada.ca
} 


\section{Introduction}

Les grands herbivores peuvent causer d'importants dommages aux écosystèmes forestiers lorsqu'ils sont présents à fortes densités (McInnes et al. 1992; Vourc'h et al. 2001; Wardle et al. 2001; McLaren et al. 2004). En plus de ses effets immédiats sur la composition et la structure des écosystèmes, l'herbivorisme soutenue entraine un long délai entre la diminution des herbivores et la réponse des communautés végétales (Pendergast et al. 2016). Par exemple, la forte abondance de cerf de Virginie (Odocoileus virginianus Zimm.) peut entrainer la quasi-disparition de la strate arbustive et le déclin de la régénération forestière (Potvin et al. 2003; Tremblay et al. 2007), voire une modification de la qualité de la litière et une diminution à long terme de la productivité des écosystèmes (Pastor et al. 1993; Harrison et Bardgett 2004; Dufresne et al. 2009).

Des mesures de restauration peuvent être entreprises pour limiter, voire inverser la transformation des écosystèmes par les grands herbivores. Par exemple, le reboisement offre l'opportunité de ramener des essences forestières dont la régénération naturelle est compromise par le broutement (de la Cretaz et Kelty 2002; Beguin et al. 2016). Toutefois, le succès des plantations dépend d'interactions complexes entre le matériel planté (e.g., type de plants), les pratiques sylvicoles (e.g., la gestion de la végétation) et des facteurs biotiques (e.g., la pression de broutement). Les dimensions initiales des plants de reboisement jouent un rôle déterminant sur leur potentiel de croissance et leur statut compétitif (Mason et al. 1996; South et Rakestraw 2004). Pour différentes essences au Québec (Canada), les plants de fortes dimensions (PFD; hauteur moyenne de 35 à $45 \mathrm{~cm}$ produits en récipient de plus de $300 \mathrm{~cm} 3$ chacun) performent généralement mieux que les plants standards sur des sites où la compétition pour les ressources est sévère (Thiffault 2004). Leur taille leur confère un avantage compétitif pour la lumière par rapport à la végétation concurrente et un potentiel de croissance plus élevé (Jobidon et al. 2003). Par ailleurs, les plants de moyennes dimensions ( $\mathrm{PMD}$; hauteur moyenne de 27 à $34 \mathrm{~cm}$ produits en récipient de $200 \mathrm{~cm}^{3}$ chacun) performent adéquatement sur des sites où le niveau de compétition est modéré (Thiffault et Roy 2011). Pour leur part, les plants de petites dimensions (PPD; hauteur moyenne de 18 à $25 \mathrm{~cm}$ produits en récipient de $110 \mathrm{~cm}^{3}$ chacun) peuvent présenter un avantage par rapport aux plants plus gros étant donné leur rapport de biomasse souterraine/aérienne élevé, lequel limite les stress lorsque les conditions environnementales sont limitantes (e.g., faible disponibilité en eau, demande évaporative de l'air élevée; Grossnickle 2000). L'effet du type de plants demeure cependant incertain dans un contexte de restauration d'écosystèmes perturbés par de fortes densités de cervidés. Des résultats à court terme (trois ans) suggèrent que les plants de moyennes dimensions constituent le meilleur compromis dans ce contexte, puisqu'ils présentent un potentiel de croissance similaire à celui de plants plus gros, tout en limitant les risques de broutement en raison de leur plus petite taille qui les rend moins visibles (Faure-Lacroix et al. 2013). Toutefois, nous ignorons quelles sont les interactions potentielles entre le type de plants, la gestion de la végétation et la présence de cerfs. En effet, le dégagement, normalement effectué pour diminuer la compétition pour les ressources, peut contribuer à augmenter le risque de broutement sur les arbres plantés en réduisant les sources de nourriture alternative (Nikula et al. 2008; Ward et al. 2008, 2018). De plus, la réaction des essences au dégagement est spécifique; elle dépend notamment de leur tolérance à l'ombre (Wagner et al. 1999).

Notre objectif était donc d'évaluer les effets simples et combinés du dégagement, du type de plants et du cerf de Virginie sur la survie et les dimensions du sapin baumier (Abies balsamea (L.) Mill.) huit ans après la mise en terre, ainsi que les effets du dégagement et du cerf et de leurs interactions sur la densité des gaules de conifères et de feuillus. Plus spécifiquement, nous visions à identifier le type de plants (petites, moyennes ou fortes dimensions) le mieux adapté aux conditions de reboisement caractérisées par une forte densité d'herbivores, et à évaluer l'effet du dégagement mécanique et du cerf sur les plants et la végétation accompagnatrice.

Nous avons prédit que les arbres soumis à un dégagement mécanique recevraient davantage de lumière et auraient des paramètres morphologiques et un taux de survie supérieurs aux arbres non dégagés (Thiffault et al. 2012), mais que ces réponses seraient dépendantes de la taille initiale des plants mis en terre (Jobidon et al. 2003). À ce titre, nous avons prédit que la taille initiale des plants influencerait leurs performances après huit ans, et que les plants de moyennes dimensions offriraient le meilleur compromis en regard de leur croissance, leur coût, et le risque de broutement qui serait diminué par rapport aux plants de fortes dimensions en raison de leur plus petite taille (Faure-Lacroix et al. 2013). Nous avons également prédit que le broutement par le cerf de Virginie jouerait un rôle négatif déterminant sur la performance des plants de sapins baumiers. Nous envisagions une survie et une croissance inférieure pour les plants croissant en présence de cerfs dans l'enclos de gestion par rapport à ceux protégés par des exclos expérimentaux (avec exclusion complète des cerfs; Ward et al. 2018), mais que les plants de moyennes dimensions seraient moins affectés par le broutement que les plants de petites ou de fortes dimensions (Faure-Lacroix et al. 2013). Finalement, nous avons prédit que le dégagement et le broutement réduiraient globalement la densité des gaules de conifères et de feuillus.

\section{Matériel et méthodes \\ Aire d'expérimentation}

L'expérience s'est déroulée sur l'île d'Anticosti (7943km² Québec, Canada, $49^{\circ} 44^{\prime} 01^{\prime \prime} \mathrm{N}, 63^{\circ} 44^{\prime} 22^{\prime \prime} \mathrm{O}$ ). Cette région se caractérise par son climat maritime qui amène en moyenne $930 \mathrm{~mm}$ de pluie chaque année; la température moyenne en janvier et en juillet est respectivement de $-10.7^{\circ} \mathrm{C}$ et $16.4^{\circ} \mathrm{C}$ (Environnement Canada 2010). La végétation est représentative du domaine bioclimatique de la sapinière à bouleau blanc (Betula papyrifera Marsh.), telle que décrite par Saucier et al. (2009), mais l'écosystème a été transformé par les cerfs introduits au $19^{\mathrm{e}}$ siècle. Suite à la disparition de tout prédateur naturel, la population de cerfs de Virginie a atteint plus de 20 cerfs $/ \mathrm{km}^{2}$ dans les dernières décennies (Potvin et Breton 2005). Le broutement chronique par le cervidé a lentement converti les peuplements de sapins et de bouleaux vers des peuplements composés en majorité d'épinettes blanches (Picea glauca (Moench) Voss; Potvin et al. 2003; Tremblay et al. 2006; Barrette et al. 2014). Le dépôt de surface est un till à texture de sable loameux (Groupe de travail sur la classification des sols 2002) dont le $\mathrm{pH}$ moyen est de 6,1. 


\section{Dispositif expérimental et traitements}

Nous avons tiré profit d'un dispositif de recherche mis en place et décrit précédemment par Faure-Lacroix et al. (2013). Le peuplement en place a été coupé par coupe avec protection de la régénération et des sols en 2004 et la construction de l'enclos de gestion, d'une superficie de $11,3 \mathrm{~km}^{2}$, a eu lieu l'année suivante. À l'intérieur de l'enclos de gestion, la pression de chasse est accentuée et vise l'atteinte d'une densité cible inférieure à 15 cerfs de Virginie par km² (Tremblay et al. 2007). Des plantations expérimentales de sapins baumiers ont été établies en juillet 2008 afin de tester les effets simples et combinés du dégagement mécanique, de la présence/ absence du cerf de Virginie et du type de plants sur la performance des sapins plantés. Ainsi, le dispositif correspond à un plan en tiroirs (split-split-plot). Les unités expérimentales (sous-sous-placettes) correspondent à des superficies adjacentes de $15 \mathrm{~m} \times 15 \mathrm{~m}$, où nous avons planté 16 plants correspondant aléatoirement à l'un des trois types de plants (PPD, PMD, PFD; Fig. 1). La hauteur et le diamètre initiaux des plants (avant la mise en terre) étaient de $18,7 \mathrm{~cm}$ et $3,8 \mathrm{~mm}$, $24,8 \mathrm{~cm}$ et $5,1 \mathrm{~mm}$, et $26,4 \mathrm{~cm}$ et $6,4 \mathrm{~mm}$ pour les PPD, PMD et PFD, respectivement (Faure-Lacroix et al. 2013). Des clôtures de $15 \mathrm{~m} \times 45 \mathrm{~m}$ excluant les cerfs de manière permanente ont été érigées et appariées à une sous-placette accessible au cerf (soumise au broutement; Fig. 1). Nous avons appliqué, en placette principale, un traitement de dégagement mécanique en 2012 sur la moitié de chacun des blocs expérimentaux (Fig. 1). Pour ce faire, nous avons procédé à la coupe de la végétation par puits de lumière (à partir du tronc du plant) à l'aide d'une débroussailleuse dans un rayon de 1,5 fois la longueur de la plus longue branche. L'ensemble des traitements étaient groupés au sein de blocs expérimentaux, lesquels ont été répétés trois fois. Au total, ce sont 576 plants de sapin baumier qui ont été mis en terre à une densité de 2500 plants/ha (espacement initial de $2 \mathrm{~m} \times 2 \mathrm{~m}$ ). Les plants ont été produits sur une période de 2 ans à partir d'une source de semences continentales dans une pépinière gouvernementale (Saint-Modeste, Québec, $48^{\circ} 26^{\prime} \mathrm{N}, 65^{\circ} 35^{\prime} \mathrm{O}$ ).

\section{Collecte de données}

Nous avons mesuré la hauteur $(\mathrm{H})$ des plants $(\mathrm{cm})$ et la longueur de la pousse terminale $(\mathrm{cm})$ des sapins en 2008, 2009, 2010, 2013 et 2015. Pour les années 2012 et 2014, nous avons estimé la hauteur en soustrayant la longueur de la pousse annuelle des années subséquentes. Nous avons mesuré le diamètre au niveau du sol $(\mathrm{D}, \mathrm{cm})$ et calculé le ratio hauteur/diamètre (ratio H/D) pour les années 2008, 2009, 2010 et 2015 . Nous avons évalué l'environnement compétitif des plants en juillet 2015 par l'estimation du recouvrement (par classe de $10 \%$ ) et la mesure de la hauteur modale (i.e., basée sur le mode, soit la hauteur représentative de la majorité des tiges) de six groupes de végétaux regroupés sur la base de leurs traits fonctionnels et de leur intérêt sylvicole : conifères, feuillus, herbacées hautes (e.g., Chamerion angustifolium, Diervilla lonicera), herbacées basses (e.g., Cornus canadensis, Linnaea borealis), graminées et framboisiers (inspiré de Balandier et al. 2006). Nous avons effectué les mesures dans des placettes circulaires centrées sur le sapin

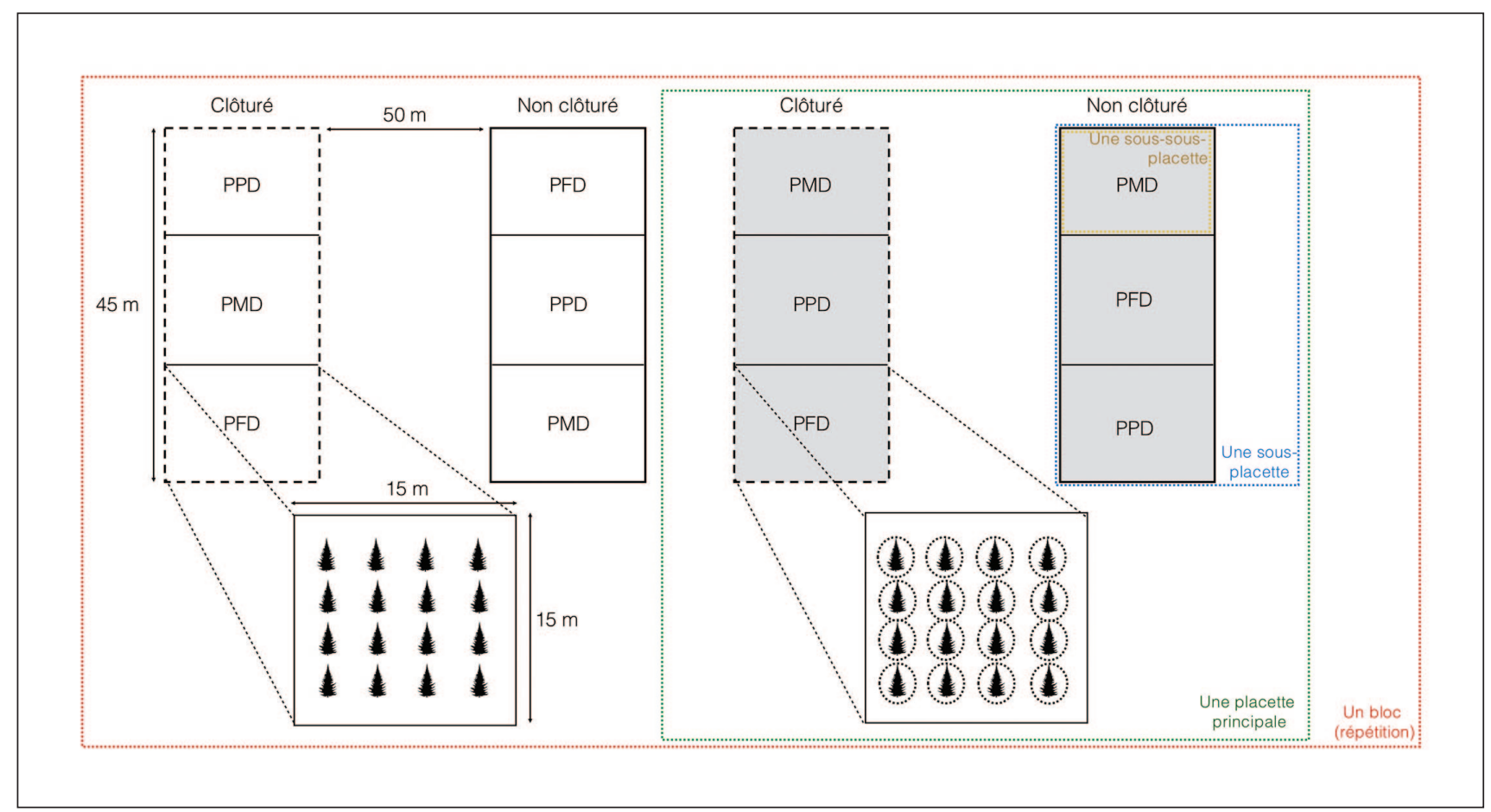

Fig. 1. Représentation d'un bloc expérimental (sur un total de trois) et de la structure hiérarchique du dispositif expérimental utilisé pour tester l'effet du type de plants, de la présence/absence du cerf de Virginie et du dégagement mécanique appliqué par pied d'arbre en 2012 (trame grise et plants encerclés) sur des plants de sapin baumier. La plantation a eu lieu en 2008 à l'île d'Anticosti (QC, Canada). Figure adaptée de Faure-Lacroix et al. (2013). PPD = plants de petites dimensions; PMD = plants de moyennes dimensions; PFD = plants de fortes dimensions. 
dont le rayon équivalait à 2 fois la longueur de la plus longue branche (rayon minimal de $1,13 \mathrm{~m}$ ). Nous avons également mesuré le rayonnement photosynthétiquement actif (\%PAR) atteignant la moitié supérieure des plants à l'aide d'un ceptomètre AccuPAR (Decagon Devices Inc., Pullman, WA, USA) selon la méthode décrite par Jobidon (1994).

À l'intérieur de chaque sous-placette $(15 \mathrm{~m} \times 15 \mathrm{~m})$, nous avons fait le décompte par espèces et par classe de hauteur $(1,3-2 \mathrm{~m}, 2-3 \mathrm{~m},>3 \mathrm{~m})$ du nombre de tiges d'essences forestières dont le diamètre à hauteur de poitrine $(1,30 \mathrm{~m}$ à partir du sol) était $\geq 1$ et $<9 \mathrm{~cm}$ (adapté de Demers 2002). Les données ont été séparées entre les conifères et les feuillus.

\section{Analyses statistiques}

Nous avons analysé les effets du dégagement mécanique, du cerf de Virginie, du type de plant et de leurs interactions sur la survie des plants en utilisant un modèle linéaire mixte généralisé correspondant à un modèle logistique (procédure GLIMMIX avec un lien logit dans le logiciel SAS, v. 9.4, SAS Institute, Cary, NC, USA) (Cao 2017). Nous avons associé les effets fixes aux différents traitements et les effets aléatoires aux blocs et aux interactions impliquant les blocs selon le modèle :

$$
\begin{aligned}
& y_{i j k h}=\mu+\beta_{i}+\tau_{j}+(\beta \tau)_{i j}+\gamma_{k}+(\tau \gamma)_{j k}+ \\
& (\beta \gamma)_{i k}+(\beta \tau \gamma)_{i j k}+\delta_{h}+(\tau \delta)_{j h}+(\gamma \delta)_{k h}+(\tau \gamma \delta)_{j k h} \\
& +(\beta \delta)_{i h}+(\beta \tau \delta)_{i j h}+\left\{\begin{array}{c}
i=1,2,3 \\
j=1,2 \\
k=1,2 \\
h=1,2,3
\end{array}\right.
\end{aligned}
$$

où $\mathrm{y}_{i j k h}$ est la mesure de la variable y dans le bloc $i$, soumise au dégagement mécanique $j$, à la presence de cerfs $k$, et au type de plant $h, \mu$ est la moyenne générale, $\beta_{i}, \tau_{j}, \gamma_{k}$ et $\delta_{h}$ sont respectivement l'effet aléatoire du bloc, l'effet fixe du d.gagement mécanique, l'effet fixe de la présence de cerfs et l'effet fixe du type de plants, et eijkh est le terme d'erreur.

Les effets des traitements sur les paramètres morphologiques (hauteur, diamètre, longueur de la flèche terminale, ratio $\mathrm{H} / \mathrm{D}$ ) et sur le pourcentage de recouvrement des végétaux ont été analysés à l'aide de modèles linéaires mixtes en utilisant la fonction lmer du progiciel lme4 v. 1.1-10 (Bates et al. 2015) disponible sous R v. 3.2.2 (R Core team 2015). Nous n'avons pas considéré le type de plant comme un effet explicatif du recouvrement et de la hauteur modale de la végétation. Nous avons restreint les analyses aux données récoltées en 2015 afin d'intégrer l'effet du dégagement mécanique appliqué en 2012. Nous avons vérifié les postulats de normalité et d'homoscédasticité à l'aide d'outils graphiques et des transformations mathématiques ont été appliquées lorsque jugées nécessaires (\%PAR : Boxcox, recouvrement de la végétation : racine carrée). Pour les traitements ayant un effet significatif, nous avons effectué des tests de comparaisons de moyenne a posteriori en utilisant la fonction lsmeans du progiciel lsmeans v. 2.23. Sauf exception, nous présentons les moyennes avec l'erreur type $( \pm \mathrm{ET})$. Nous avons utilisé un seuil alpha de 0,05 pour déclarer un effet significatif et considéré des valeurs se situant entre 0,05 et 0,10 comme dénotant une tendance.

\section{Résultats}

\section{Survie et morphologie des plants}

Les traitements n'influencent pas la survie des plants huit ans après leur mise en terre (Tableau 1). Le taux de survie moyen des plants est de $88 \% \pm 5$.

Huit ans après la plantation, la hauteur et le diamètre des plants sont significativement influencés par le type de plants (Tableau 1). Nous n'observons aucune différence de hauteur, de diamètre ou de ratio entre ces variables pour les plants de fortes et de moyennes dimensions (Tableau 1, Fig. 2, comparaison a posteriori; hauteur : $\mathrm{t}_{16}=0,7 ; p=0,49$; diamètre : $\mathrm{t}_{16}=0,53 ; p=0,60$; ratio H/D: $\mathrm{t}_{16}=0,32 ; p=0,75$ ) alors que les plants de petites dimensions présentent toujours une hauteur, un diamètre et un ratio H/D inférieur (Tableau 1, Fig. 2). Le ratio $H / D$ est significativement plus élevé pour les arbres ayant crû en absence de cerf (46 \pm 1$)$ que pour ceux soumis à la pression de broutement $(42 \pm 1)$ (Tableau 1). Aucun des traitements n'influence significativement la longueur de la flèche terminale (Tableau 1).

\section{Végétation accompagnatrice et lumière}

Nous observons un recouvrement significativement inférieur des herbacées hautes dans les placettes exposées au broutement $(5 \% \pm 4)$ par rapport à celui mesuré dans les placettes protégées des cerfs $(26 \% \pm 10)$, mais uniquement en absence de dégagement (interaction significative; Tableau 2). Nous observons une tendance statistique pour une hauteur modale moindre des herbacées hautes dans les placettes non dégagées soumises au broutement $(36 \pm 5 \mathrm{~cm})$ par rapport à celles dégagées non soumises au broutement $\left(82 \pm 8 \mathrm{~cm} ; \mathrm{t}_{2}=-4.8\right.$; $p=0,10)$. La hauteur modale des feuillus baisse en présence de cerfs (Tableau 2; $92 \pm 41 \mathrm{~cm}$ en présence de cerfs versus $201 \pm 60 \mathrm{~cm}$ dans les exclos).

Les traitements de dégagement et d'exclusion du broutement n'influencent pas l'abondance des gaules de conifères (Tableau 3). Les gaules de conifères de 1,3-2 $\mathrm{m}$ et de 2-3 m ont une densité similaire dépassant 1500 tiges/ha $\left(\mathrm{t}_{16}=-1,9\right.$; $p=0,17$ ), alors que les tiges de $3 \mathrm{~m}$ et plus ont une densité de 302 tiges/ha \pm 135 . L'exclusion des cerfs tend à influencer la densité des tiges décidues, mais seulement pour la classe de hauteur de $3 \mathrm{~m}$ et plus (Tableau 3; 503 tiges/ha \pm 227 dans les exclos versus 1 tige/ha $\left.\pm 11, \mathrm{t}_{4}=-4,6 ; p=0,06\right)$. Nous ne décelons aucune différence significative associée à la présence de cerfs pour les tiges décidues des classes de $1,3-2 \mathrm{~m} \mathrm{(t_{4 } =}$ $0,32 ; p=1,00)$ et de $2-3 \mathrm{~m}\left(\mathrm{t}_{4}=-2,9 ; p=0,21\right)$.

Le \%PAR est significativement plus élevé en présence de cerfs $(74 \% \pm 2)$ que dans les exclos (Tableau $1 ; 66 \% \pm 2 ; \mathrm{t}_{4}=$ $3.5 ; p=0.02$ ). Trois ans après son application, le dégagement mécanique n'a pas d'influence sur le pourcentage de lumière atteignant la moitié supérieure des plants. Les types de plants reçoivent également une quantité de lumière équivalente entre eux.

\section{Discussion}

Notre objectif était d'évaluer les effets du dégagement mécanique sur la survie et les paramètres morphologiques du sapin baumier en interaction avec le type de plants et la pression de broutement par le cerf de Virginie en enclos de gestion soumis à la chasse sportive. Nous visions également à évaluer comment le dégagement et le cerf influençaient la 
Tableau 1. Résumé des résultats des modèles testant les effets du dégagement mécanique (DM), de l'exclusion du cerf de Virginie $(E X)$ et des types de plants $(T)$ sur la survie (modèle linéaire mixte généralisé, distribution logistique) et les paramètres morphologiques (modèle linéaire mixte) de plants de sapin baumier, 8 saisons de croissance après leur mise en terre. Les plants ont été mis en terre en 2008 à lî́le d'Anticosti (Canada). La longueur de la flèche correspond à la pousse annuelle de la $8^{e}$ saison de croissance. $\mathrm{dl}=$ degrés de liberté (numérateur, dénominateur). \%PAR = rayonnement photosynthétiquement actif atteignant la moitié supérieure des plants.

\begin{tabular}{|c|c|c|c|c|c|c|c|c|c|c|c|c|c|}
\hline \multicolumn{2}{|c|}{$\begin{array}{l}\text { Sources de } \\
\text { variation (fixes) }\end{array}$} & \multicolumn{2}{|c|}{ Survie } & \multicolumn{2}{|c|}{ Hauteur } & \multicolumn{2}{|c|}{$\begin{array}{l}\text { Diamètre au } \\
\text { niveau du sol }\end{array}$} & \multicolumn{2}{|c|}{$\begin{array}{l}\text { Longueur de } \\
\text { la flèche }\end{array}$} & \multicolumn{2}{|c|}{$\begin{array}{c}\text { Ratio } \\
\text { Hauteur/Diamètre }\end{array}$} & \multicolumn{2}{|c|}{$\%$ PAR } \\
\hline & dl & F & $p$ & F & $p$ & F & $p$ & F & $p$ & F & $p$ & F & $p$ \\
\hline $\begin{array}{l}\text { Dégagement } \\
\text { mécanique } \\
\text { (DM) }\end{array}$ & 1,2 & 0,31 & 0,63 & 1,25 & 0,38 & 0,03 & 0,89 & 0,15 & 0,73 & 16,96 & 0,05 & 3,03 & 0,22 \\
\hline $\begin{array}{l}\text { Exclusion } \\
\text { (EX) }\end{array}$ & 1,4 & 0,49 & 0,52 & 1,38 & 0,31 & 0,02 & 0,89 & 0,09 & 0,78 & 9,82 & 0,04 & 15,11 & 0,02 \\
\hline $\mathrm{DM} \times \mathrm{EX}$ & 1,4 & 1,45 & 0,29 & 0,14 & 0,73 & 0,03 & 0,87 & 0,20 & 0,68 & 3,42 & 0,14 & 2,32 & 0,20 \\
\hline Types (T) & 2,16 & 2,09 & 0,16 & 5,71 & 0,01 & 11,18 & $<0,01$ & 0,84 & 0,45 & 4,00 & 0,04 & 1,23 & 0,32 \\
\hline $\mathrm{DM} \times \mathrm{T}$ & 2,16 & 2,70 & 0,10 & 2,16 & 0,15 & 2,23 & 0,14 & 0,95 & 0,41 & 1,16 & 0,34 & 0,16 & 0,85 \\
\hline $\mathrm{EX} \times \mathrm{T}$ & 2,16 & 0,25 & 0,78 & 0,82 & 0,46 & 1,83 & 0,19 & 1,38 & 0,28 & 1,40 & 0,27 & 1,41 & 0,27 \\
\hline $\mathrm{DM} \times \mathrm{EX} \times \mathrm{T}$ & 2,16 & 0,13 & 0,88 & 0,24 & 0,79 & 0,44 & 0,65 & 0,13 & 0,88 & 1,20 & 0,33 & 0,62 & 0,55 \\
\hline
\end{tabular}

densité des gaules. Trois ans après l'application du traitement de dégagement (huit ans après la mise en terre), nous n'avons mesuré aucun effet notable du dégagement sur la survie et les paramètres morphologiques des plants de sapin baumier. Nos résultats indiquent ainsi que le dégagement mécanique par pied d'arbre offre peu d'avantages sylvicoles dans un contexte de restauration du sapin baumier dans ces conditions.

Le dégagement mécanique s'est avéré efficace pour favoriser la croissance des conifères dans d'autres contextes (e.g., Jobidon et Charrette 1997). Par exemple, en diminuant le recouvrement des espèces concurrentes de manière temporaire, le dégagement résulte généralement en une productivité accrue, tant à l'échelle de l'arbre qu'à celle du peuplement pour des plantations d'épinettes blanche ou noire ( $P$. glauca, P. mariana (Mill.) BSP; Urli et al. 2018). Cependant, les informations sur la réaction du sapin baumier au dégagement mécanique demeurent rares, cette essence étant peu reboisée dans un contexte de production forestière au Québec. Notre étude a démontré que la réponse du sapin à la maîtrise de la végétation dans le contexte des plantations sur Anticosti est différente de celles attendues pour les épinettes. Ceci s'explique, en partie, par l'absence d'influence du traitement de dégagement par pied d'arbres sur le recouvrement et la hauteur de la végétation concurrente, la densité des gaules de feuillus et de conifères et la proportion de pleine lumière atteignant la moitié supérieure des plants mis en terre. La modalité de dégagement par pied d'arbre a été choisie, dans ce contexte, afin de conserver une partie du brout alternatif disponible aux cerfs, de manière à ne pas transférer entièrement la pression de broutement sur les arbres plantés (Ward et al. 2018). Or, le maintien de bandes de végétation entre les plants en raison du rayon de traitement relativement court autour de ceux-ci a certainement accéléré le ré-envahissement des zones dégagées par les espèces concurrentes. Également, les tiges résineuses et décidues qui n'ont pas été coupées ont pu croître en hauteur et leur houppier ont pu s'élargir et ainsi occuper l'espace laissé libre par la coupe des tiges dans l'environnement immédiat des plants. La diminution de la compétition pour les ressources n'aurait donc pas été d'un niveau ou d'une durée suffisante pour stimuler la croissance des sapins baumiers plantés.

À l'instar des observations à court terme de Faure-Lacroix et al. (2013), nous avons constaté que les plants de moyennes dimensions ont atteint des dimensions similaires à celles des plants de fortes dimensions. Les PMD ont maintenu des taux de croissance en hauteur et en diamètre similaires à ceux des PFD entre la $3^{\mathrm{e}}$ et les $8^{\mathrm{e}}$ saisons de croissance, et ce, sans égard au niveau de compétition (absence d'interaction entre le type de plants et le traitement de dégagement). L'écart entre les plants de forts gabarits (PMD et PFD) et ceux de petites dimensions (PPD) s'est par ailleurs accru entre la $3^{\mathrm{e}}$ et la $8^{\mathrm{e}}$ saison de croissance, et ce, tant en hauteur qu'en diamètre. Ces résultats correspondent à notre prédiction; ils supportent donc l'hypothèse que les plants de moyennes dimensions ont une taille initiale suffisante pour leur conférer un avantage compétitif par rapport à la végétation concurrente et qu'il n'y a pas d'avantage biologique à une taille supérieure au moment de la mise en terre (Jobidon et al. 2003). Les coûts de production, de transport et de plantation sont moins élevés pour les plants de moyennes dimensions que pour les plants de fortes dimensions (Thiffault 2004). Dans ce contexte, et étant donné que les PMD présentent des performances simi- 
A

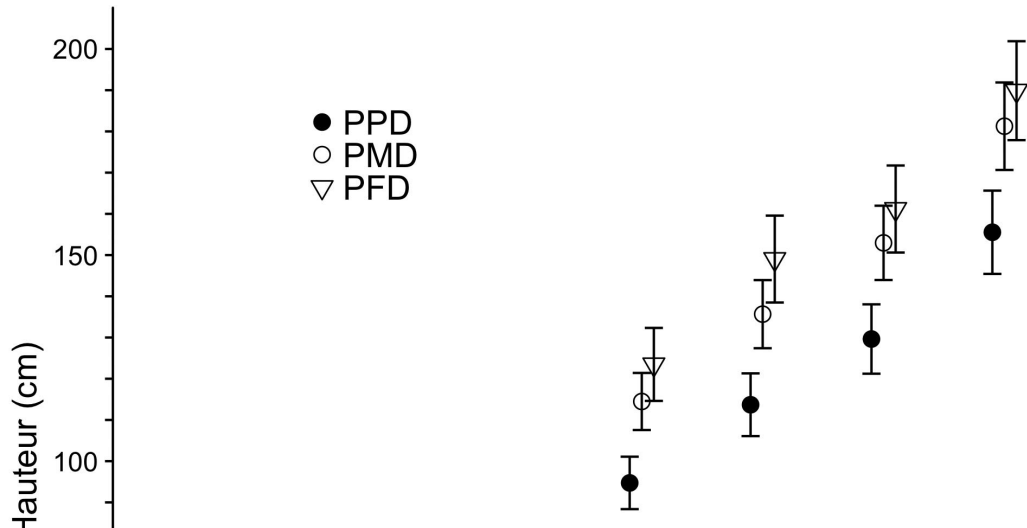

B

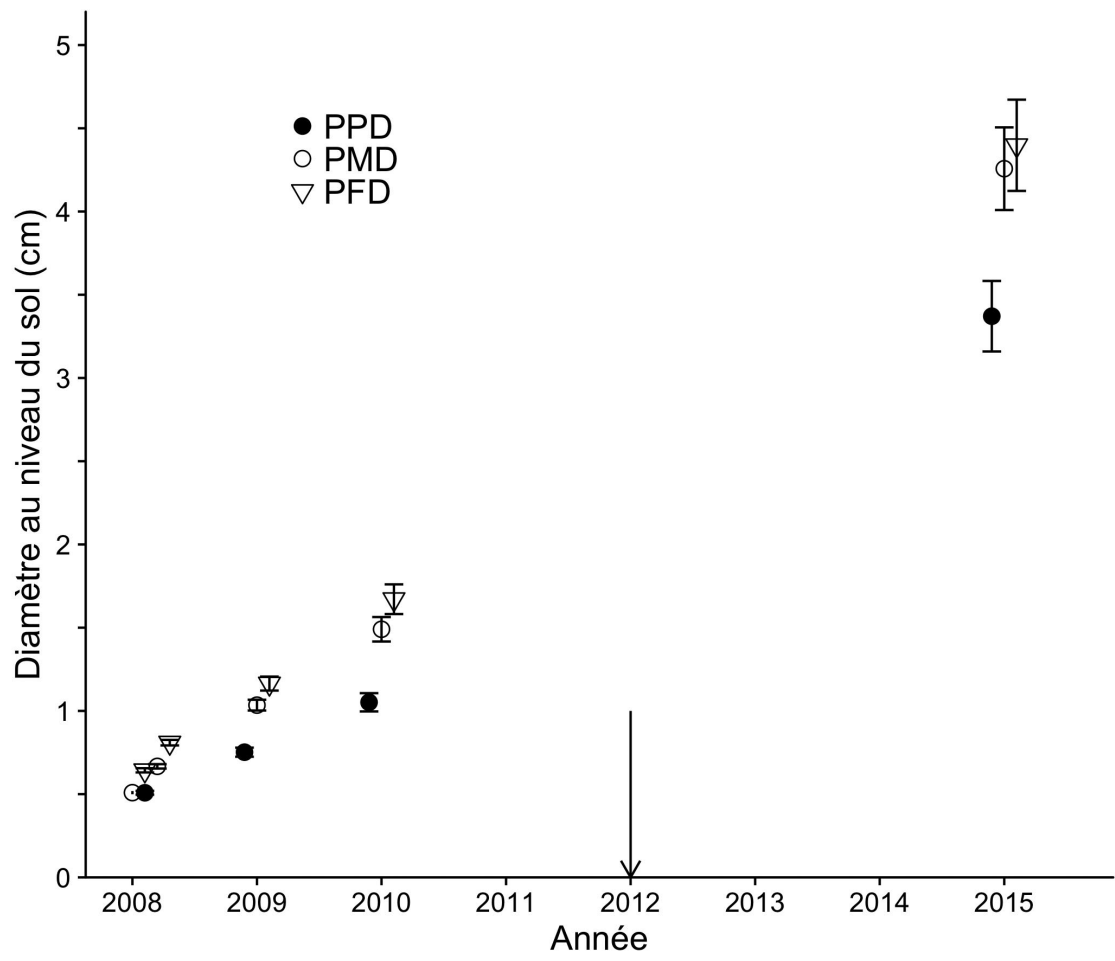

Fig. 2. Hauteur (A) et diamètre au niveau du sol (B) de trois types de plants de sapin baumier dans une plantation expérimentale de lî̀le d’Anticosti (QC, Canada). Un dégagement mécanique par pied d'arbre a été réalisé en 2012 (indiqué par la flèche). Les données correspondent aux moyennes brutes \pm IC $95 \%$. Les analyses ont été réalisées sur les données récoltées en 2015 ; les autres données sont présentées pour fins d'information seulement. PPD = plants de petites dimensions; PMD = plants de moyennes dimensions; PFD = plants de fortes dimensions. 
Tableau 2. Résumé des résultats des modèles linéaires mixtes testant les effets interactions sur le recouvrement et la hauteur modale de différents groupes de végétaux. Les mesures ont été prises en 2015 dans des parcelles circulaires centrées sur des plants de sapin baumier mis en terre en 2008 à l'île d'Anticosti [Canada]. Les degrés de liberté au numérateur étaient de 1 pour tous les effets ; les degrés de liberté aux dénominateurs étaient de 2, 4, et 4 pour les effets DM, EX, et DM×EX, respectivement.

\begin{tabular}{|c|c|c|c|c|c|}
\hline $\begin{array}{l}\text { Groupe } \\
\text { d'espèces }\end{array}$ & Variable réponse & & $\begin{array}{l}\text { Dégagement } \\
\text { mécanique } \\
\text { (DM) }\end{array}$ & $\begin{array}{c}\text { Exclusion } \\
\text { (EX) }\end{array}$ & $\mathrm{DM} \times \mathrm{EX}$ \\
\hline \multirow{4}{*}{ Framboiser } & \multirow{2}{*}{$\%$ recouvrement } & $\mathrm{F}$ & 3,82 & 0,05 & 0,00 \\
\hline & & $p$ & 0,19 & 0,84 & 0,97 \\
\hline & \multirow{2}{*}{ Hauteur modale } & $\mathrm{F}$ & 3,05 & 0,90 & 0,60 \\
\hline & & $p$ & 0,22 & 0,40 & 0,48 \\
\hline \multirow{4}{*}{ Feuillus } & \multirow{2}{*}{$\%$ recouvrement } & $\mathrm{F}$ & 0,64 & 4,00 & 5,72 \\
\hline & & $p$ & 0,51 & 0,12 & 0,08 \\
\hline & \multirow{2}{*}{ Hauteur modale } & $\mathrm{F}$ & 0,14 & 20,00 & 0,07 \\
\hline & & $p$ & 0,75 & 0,01 & 0,81 \\
\hline \multirow{4}{*}{ Conifères } & \multirow{2}{*}{$\%$ recouvrement } & $\mathrm{F}$ & 0,85 & 1,31 & 1,24 \\
\hline & & $p$ & 0,45 & 0,32 & 0,33 \\
\hline & \multirow{2}{*}{ Hauteur modale } & $\mathrm{F}$ & 0,03 & 0,02 & 0,09 \\
\hline & & $p$ & 0,87 & 0,90 & 0,78 \\
\hline \multirow{4}{*}{$\begin{array}{l}\text { Herbacées } \\
\text { hautes }\end{array}$} & \multirow{2}{*}{$\%$ recouvrement } & $\mathrm{F}$ & 0,00 & 84,05 & 15,17 \\
\hline & & $p$ & 0,99 & $<0,01$ & 0,02 \\
\hline & \multirow{2}{*}{ Hauteur modale } & $\mathrm{F}$ & 0,00 & 354,64 & 14,56 \\
\hline & & $p$ & 0,97 & $<0,01$ & 0,02 \\
\hline \multirow{4}{*}{$\begin{array}{l}\text { Herbacées } \\
\text { basses }\end{array}$} & \multirow{2}{*}{$\%$ recouvrement } & $\mathrm{F}$ & 0,17 & 0,45 & 0,78 \\
\hline & & $p$ & 0,72 & 0,54 & 0,43 \\
\hline & \multirow{2}{*}{ Hauteur modale } & $\mathrm{F}$ & - & - & - \\
\hline & & $p$ & - & - & - \\
\hline \multirow{4}{*}{ Graminées } & \multirow{2}{*}{$\%$ recouvrement } & $\mathrm{F}$ & 3,12 & 5,58 & 4,33 \\
\hline & & $p$ & 0,22 & 0,08 & 0,11 \\
\hline & \multirow{2}{*}{ Hauteur modale } & $\mathrm{F}$ & 0,01 & 0,00 & 1,98 \\
\hline & & $p$ & 0,95 & 0,96 & 0,23 \\
\hline
\end{tabular}
du dégagement mécanique (DM), de l'exclusion du cerf de Virginie (EX) et leurs

rapide de la pression de broutement qui suit l'enlèvement des clôtures (i.e., le passage d'une densité de cerfs $<15$ individus $/ \mathrm{km}^{2}$ à une densité $\geq 20$ individus $/ \mathrm{km}^{2}$ ). Si la nourriture alternative n'est pas suffisante, nous doutons que les PPD survivront à cette prochaine étape du scénario sylvicole; le suivi à plus long terme du dispositif expérimental permettra de vérifier cette prédiction. Contrairement à notre prédiction, le cerf de Virginie n'a pas eu d'effet marqué sur la survie et les variables morphologiques des plants mis en terre. De plus, le cerf ne semble pas avoir brouté davantage les PFD que les autres types de plants. Ceci est probablement associé au fait que, bien que le diamètre initial des PFD était plus élevé que celui des autres types de plants, leur hauteur était similaire à celle des PMD au moment de leur mise en terre (Faure-Lacroix et al. 2013). Nous concluons que la réduction de la densité de cerfs par la chasse sportive dans l'enclos de gestion a permis de diminuer le broutement à un point tel que, 8 ans après la mise en terre, la pression de broutement n'est plus un facteur limitant la croissance de la régénération dans ce type d'écosystème. L'absence d'interaction entre l'exclusion des cerfs et le dégagement mécanique suggère par ailleurs que la coupe de la végétation à proximité des plants de sapin n'a pas accentué la vulnérabilité des arbres au broutement.

En présence de cerf, nous avons observé une diminution marquée de la densité des feuillus de $3 \mathrm{~m}$ et plus de hauteur. Également, les cerfs ont eu tendance à diminuer la hauteur des herbacées hautes autant, voire davantage que le traitement de dégagement $(36 \mathrm{~cm}$ versus $82 \mathrm{~cm}$, mais cette différence n'était pas statistiquement significative). Les grands

laires à celles des plants de fortes dimensions tout en étant plus performants que les plants de petites dimensions, ils apparaissent comme le meilleur choix de type de plants à utiliser pour ces efforts restauration. De manière plus générale, ces résultats supportent que le choix du type de plants dans les projets de restauration écologique doit être adapté aux objectifs du reboisement et tenir compte des contraintes biotiques et abiotiques à l'établissement et la croissance (target plant concept, sensu Dumroese et al. 2016).

Par ailleurs, les plants de petites dimensions ont atteint, en huit ans, une hauteur tout juste suffisante pour limiter le broutement de leur flèche terminale (une hauteur qui se situe à environ $140 \mathrm{~cm}$; Brousseau et al. 2017). L'espérance de vie des enclos de gestion est estimée à environ 10-12 ans (Beaupré et al. 2004; G. Laprise, comm. pers.); il est donc critique que les arbres plantés possèdent une hauteur et une biomasse foliaire suffisantes pour survivre à l'augmentation herbivores peuvent en effet diminuer la compétition pour les ressources et ainsi créer un avantage compétitif apparent pour les espèces qui leur sont moins attirantes (Rooney et Waller 2003; Serra et al. 2014). Par exemple, une densité d'orignaux inférieure à 1,5 individus $/ \mathrm{km}^{2}$ réduit significativement la compétition par les feuillus envers l'épinette blanche et le sapin baumier (McLaren et al. 2009; Posner et Jordan 2002). La diminution du couvert et de la hauteur modale des herbacées hautes, combinée avec la diminution de la densité de feuillus de plus de $3 \mathrm{~m}$ pourrait expliquer l'augmentation du niveau de lumière que nous avons mesuré dans la moitié supérieure des plants ayant crû en présence de cerfs. Cette amélioration des conditions de lumière n'a toutefois pas résulté en des gains de croissance pour les plants, cette ressource n'étant probablement pas limitante pour le sapin baumier aux conditions mesurées dans notre étude (66 à 74 $\%)$. En effet, le sapin baumier est une essence très tolérante à 
Tableau 3. Résumé des résultats des modèles linéaires mixtes testant les effets du dégagement mécanique (DM), de l'exclusion du cerf de Virginie (EX), de la classe de hauteur et leurs interactions sur la densité de gaules à l'hectare des conifères et des feuillus dans une plantation de sapin baumier à l'île d'Anticosti (Canada). Les mesures ont été prises à l'intérieur de sous-parcelles $15 \mathrm{~m} \times 45 \mathrm{~m}$ excluant les cerfs de manière permanente auxquelles étaient appariées des sousparcelles accessibles aux cerfs. $\mathrm{dl}=$ degrés de liberté (numérateur, dénominateur]

\begin{tabular}{|c|c|c|c|c|c|}
\hline \multirow[b]{2}{*}{ Sources de variation (fixes) } & \multirow[b]{2}{*}{ dl } & \multicolumn{2}{|c|}{ Conifères } & \multicolumn{2}{|c|}{ Feuillus } \\
\hline & & $\mathbf{F}$ & $p$ & $\mathbf{F}$ & $p$ \\
\hline $\begin{array}{l}\text { Dégagement } \\
\text { mécanique (DM) }\end{array}$ & 1,2 & 1,06 & 0,41 & 1,47 & 0,35 \\
\hline Exclusion (EX) & 1,4 & 1,79 & 0,25 & 17,13 & 0,01 \\
\hline $\mathrm{DM} \times \mathrm{EX}$ & 2,16 & 1,85 & 0,25 & 0,37 & 0,57 \\
\hline Classe de hauteur $(\mathrm{CH})$ & 1,4 & 34,33 & $<0,01$ & 2,14 & 0,15 \\
\hline $\mathrm{DM} \times \mathrm{CH}$ & 2,16 & 0,09 & 0,91 & 0,35 & 0,71 \\
\hline $\mathrm{EX} \times \mathrm{CH}$ & 2,16 & 1,21 & 0,32 & 6,22 & 0,01 \\
\hline $\mathrm{DM} \times \mathrm{EX} \times \mathrm{CH}$ & 2,16 & 0,73 & 0,50 & 0,47 & 0,64 \\
\hline
\end{tabular}

des populations de cervidés. Par exemple, ils indiquent que le choix du type de plants pour le reboisement, souvent dicté par des considérations opérationnelles, devrait prendre en compte les risques de broutement. De plus, nos résultats illustrent l'importance d'une approche intégrée de gestion de la végétation, laquelle doit non seulement être basée sur une connaissance des espèces concurrente, mais également sur les dommages potentiels à la régénération par la grande faune.

\section{Remerciements}

Nous remercions Julie Faure-Lacroix, Jacques Carignan, Govinda Saint-Pierre et Simon Désalliers pour leur contribution à l'établissement, aux mesurages initiaux et à l'entretien du dispositif expérimental, de même qu'Evelyne Lemay qui a collaboré à la prise des mesures en 2015. Les commentaires constructifs de deux réviseurs externes ont permis d'améliorer une version précédente de ce texte; nous leur en sommes reconnaissants. Ce projet a été financé par le Conseil de recherches en sciences naturelles et en génie du

l'ombre (Frank 1990; Humbert et al. 2007); elle établit généralement une banque de semis qui survivent plusieurs décennies dans le sous-bois des peuplements matures (Parent et Ruel 2002).

\section{Conclusion}

L'augmentation des populations de grands herbivores est un phénomène multicontinental; ses impacts sur les écosystèmes forestiers sont observés dans plusieurs biomes (Fuller et Gill 2001; Ward 2005; Takatsuki 2009; Austrheim et al. 2011; Nuttle et al. 2013). La sylviculture offre l'opportunité de réduire, voire d'inverser les impacts des grands herbivores sur la dynamique des écosystèmes (Beguin et al. 2016), mais les modalités des traitements sylvicoles jouent un rôle significatif dans le succès des travaux de restauration (Moore et al. 1999). Nos résultats, issus d'une expérience permettant de distinguer les effets simples et combinés du type de plants, de la pression de broutement et du dégagement mécanique sur la survie et la croissance du sapin baumier, nous indiquent que les effets principaux de ces facteurs dominent sur leurs interactions. Nous concluons : 1) que les plants de moyennes dimensions offrent le meilleur compromis de taille pour la restauration des sapinières boréales soumises au broutement par les grands herbivores; 2) que le dégagement mécanique n'est pas justifié d'un point de vue sylvicole dans ce contexte; et, 3) que la chasse sportive en enclos permet de réduire la pression de broutement suffisamment pour que la survie et la croissance des plants ne soit plus significativement affectées, tout en permettant de diminuer la compétition par les feuillus.

Au-delà du contexte spécifique de l'île d'Anticosti, nos résultats fournissent des pistes pour l'aménagement et la sylviculture dans les régions soumises à une augmentation
Canada, Solifor Anticosti, la Société des établissements de plein air du Québec ainsi que le Ministère des Forêts, de la Faune et des Parcs par l'entremise de la Chaire de recherche industrielle CRSNG en aménagement intégré des ressources de l'île d'Anticosti (S. Côté) et la collaboration de la Direction de la recherche forestière.

\section{Bibliographie}

Austrheim, G., E.J. Solberg et A. Mysterud. 2011. Spatio-temporal variation in large herbivore pressure in Norway during 1949-1999: Has decreased grazing by livestock been countered by increased browsing by cervids? Wildlife Biol.7(3): 286-298. doi:10.2981/10-038. Balandier, P., C. Collet, J.H. Miller, P.E. Reynolds et S.M. Zedaker. 2006. Designing forest vegetation management strategies based on the mechanisms and dynamics of crop tree competition by neighbouring vegetation. Forestry 79(1): 3-27. doi:10.1093/ forestry/cpi056.

Barrette, M., L. Bélanger, L. De Grandpré et J.-C. Ruel. 2014. Cumulative effects of chronic deer browsing and clear-cutting on regeneration processes in second-growth white spruce stands. Forest Ecol. Manag.329: 69-78. doi:10.1016/j.foreco.2014.06.020.

Bates, D., M. Mächler, B.M. Bolker et S.C. Walker. 2015. Fitting linear mixed-effects models using lme4. J. Stat. Softw. 67(1): 1-48. doi:10.18637/jss.v067.i01.

Beaupré, P., C. Bédard, C. Dufour, A. Gingras, C. Malenfant et F. Potvin. 2004. Plan général d'aménagement intégré des ressources du milieu forestier de l'île d'Anticosti. Produits forestiers Anticosti inc., Ministère des Ressources naturelles, de la Faune et des Parcs, Société de la faune et des parcs du Québec. Québec, QC.

Beguin, J., J.-P. Tremblay, N. Thiffault, D. Pothier et S.D. Côté. 2016. Management of forest regeneration in boreal and temperate deer-forest systems: Challenges, guidelines and research gaps. Ecosphere 7(10): e01488. doi:10.1002/ecs2.1488.

Brousseau, M., N. Thiffault, J. Beguin, V. Roy et J.-P. Tremblay. 2017. Deer browsing outweighs the effects of site preparation and mechanical release on balsam fir seedlings performance: Implica- 
tions to forest management. Forest Ecol. Manag. 405: 360-366. doi:10.1016/j.foreco.2017.09.024.

Cao, Q.V. 2017. Evaluation of methods for modeling individual tree survival. Forest Sci. 63(4): 356-361. doi:10.5849/forsci.2016-001.

de la Cretaz, A.L. et M.J. Kelty. 2002. Development of tree regeneration in fern-dominated forest understories after reduction of deer browsing. Restor. Ecol. 10(2) : 416-426. doi:10.1046/j.1526100X.2002.02037.x.

Demers, D. 2002. Normes d'inventaire forestier. Placettes-échantillons temporaires. Peuplements de moins de $7 \mathrm{~m}$ de hauteur. Direction des inventaires forestiers. Ministère des Ressources naturelles. Québec, QC.

Dufresne, M., R.L. Bradley, J.-P. Tremblay, M. Poulin et S. Pellerin. 2009. Clearcutting and deer browsing intensity interact in controlling nitrification rates in forest floor humus. Ecosci. 16(3): 361-368. doi:10.2980/16-3-3267.

Dumroese, K.R., T.D. Landis, J.R. Pinto, D.L. Haase, K.W. Wilkinson et A.S. Davis. 2016. Meeting forest restoration challenges: Using the target plant concept. Reforesta 1(1): 37-52. doi:10.21750/REFOR.1.03.3.

Environnement Canada. 2010. Normales climatiques canadiennes 1981-2010. Disponible à http://climate.weather.gc.ca/climate_normals/index_e.html [page consultée le 26 août 2016].

Faure-Lacroix, J., J.-P. Tremblay, N. Thiffault et V. Roy. 2013. Stock type performance in addressing top-down and bottom-up factors for the restoration of indigenous trees. Forest Ecol. Manag. 307: 333-340. doi:10.1016/j.foreco.2013.07.031.

Frank, R.M. 1990. Abies balsamea L. Mill. - Balsam fir. Dans Silvics of North America: Volume 1, Conifers. Édité par Burns, R.M. et B.H. Honkala. Forest Service, United States Department of Agriculture, Washington, D.C. pp. 26-35.

Fuller, R.J. et R.M.A. Gill. 2001. Ecological impacts of increasing numbers of deer in British woodland. Forestry 74(3): 193-199.

Grossnickle, S.C. 2000. Ecophysiology of Northern Spruce Species. The Performance of Planted seedlings. NRC Research Press, Ottawa, ON.

Groupe de travail sur la classification des sols. 2002. Le système canadien de classification des sols. Agriculture et Agro-Alimentaire Canada. Publication 1646. 196 p.

Harrison, K.A. et R.D. Bardgett. 2004. Browsing by red deer negatively impacts on soil nitrogen availability in regenerating native forest. Soil Biol. Biochem. 36(1): 115-126. doi:10.1016/j.soilbio. 2003.08.022.

Humbert, L., D. Gagnon, D. Kneeshaw et C. Messier. 2007. A shade tolerance index for common understory species of northeastern North America. Ecol. Indic. 7(1): 195-207. doi:10.1016/j.ecolind.2005.12.002.

Jobidon, R. 1994. Light threshold for optimal black spruce (Picea mariana) seedling growth and development under brush competition. Can. J. For. Res. 24(8): 1629-1635. doi:10.1139/x94-211.

Jobidon, R. et L. Charette. 1997. Effets, après 10 ans, du dégagement manuel simple ou répété et de la période de coupe de la végétation de compétition sur la croissance de l'épinette noire en plantation. Can. J. For. Res. 27(12): 1979-1991. doi:10.1139/x97-166.

Jobidon, R., V. Roy et G. Cyr. 2003. Net effect of competing vegetation on selected environmental conditions and performance of four spruce seedling stock sizes after eight years in Québec (Canada). Ann. For. Sci. 60(7): 691-699. doi:10.1051/forest: 2003063.

Mason, E.G., D.B. South et Z. Weizhong. 1996. Performance of Pinus radiata in relation to seedling grade, weed control, and soil cultivation in the central north island of New Zealand. N. Z. J. For. Sci. 26(1/2): 173-183.

McInnes, P., R.J. Naiman, J. Pastor et Y. Cohen. 1992. Effects of moose browsing on vegetation and litter of the boreal forest, Isle Royale, Michigan, USA. Ecology 73(6): 2059-2075. doi:10.2307/ 1941455.
McLaren, B.E., L. Hermanutz, J. Gosse, B. Collet et C. Kasimos. 2009. Broadleaf competition interferes with balsam fir regeneration following experimental removal of moose. Forest Ecol. Manag. 257: 1395-1404. doi:10.1016/j.foreco.2008.12.009.

McLaren, B.E., B.A. Roberts, N. Djan-Chekar et K.P. Lewis. 2004. Effects of overabundant moose on the Newfoundland landscape. Alces 40: 45-59.

Moore, N.P., J.D. Hart et S.D. Langton. 1999. Factors influencing browsing by fallow deer Dama dama in young broad-leaved plantations. Biol. Conserv. 87(2): 255-260. doi:10.1016/S0006-3207 (98)00055-X.

Nikula, A., V. Hallikainen et R. Jalkanen. 2008. Modelling the factors predisposing scots pine to moose damage in artificially regenerated sapling stands in Finnish Lapland. Silva Fenn. 42(4): 587-603.

Nuttle, T., A.A. Royo, M. Beth Adams et W.P. Carson. 2013. Historic disturbance regimes promote tree diversity only under low browsing regimes in eastern deciduous forest. Ecol. Mono. 83(1): 317. doi:10.1890/11-2263.1.

Parent, S. et J.-C. Ruel. 2002. Chronologie de la croissance chez des semis de sapin baumier (Abies balsamea (L.) Mill.) après une coupe à blanc avec protection de la régénération. For. Chron. 78(6): 876885.

Pastor, J., B. Dewey, R.J. Naiman, P.F. McInnes et Y. Cohen. 1993. Moose browsing and soil fertility in the boreal forests of Isle Royale National Park. Ecology 74(2): 467-480. doi:10.2307/ 1939308.

Pendergast, T.H., S.M. Hanlon, Z.M. Long, A.A. Royo et W.P. Carson. 2016. The legacy of deer overabundance: Long-term delays in herbaceous understory recovery. Can. J. For. Res. 46(3): 362-369. doi:10.1139/cjfr-2015-0280.

Posner, S.D. et P.A. Jordan. 2002. Competitive effects on plantation white spruce saplings from shrubs that are important browse for moose. Forest Sci. 48(2): 283-289.

Potvin, F. et L. Breton. 2005. Testing two aerial survey techniques on deer in fenced enclosures: Visual double-counts and thermal infrared sensing. Wildlife Soc. B. 33(1): 317-325.

Potvin, F., P. Beaupré et G. Laprise. 2003. The eradication of balsam fir stands by white-tailed deer on Anticosti Island, Québec: A 150-year process. Ecoscience 10: 487-495.

R Core Team. 2015. R: A language and environment for statistical computing. R Foundation for Statistical Computing, Vienna, Austria. Rooney, T.P. et D.M. Waller. 2003. Direct and indirect effects of white-tailed deer in forest ecosystems. Forest Ecol. Manag. 181: 165-176. doi:10.1016/s0378-1127(03)00130-0.

Saucier, J., A. Robitaille et P. Grondin. 2009. Cadre bioclimatique du Québec. Dans Manuel de foresterie. Ordre des ingénieurs forestiers du Québec, Québec, Québec. pp. 186-205.

Serra, R., C. Opio et D.P. Khasa. 2014. Sheep vegetation management for controlling competing vegetation in young conifer plantations in the central interior of British Columbia, Canada. Agroforest. Syst. 88(4): 569-578. doi:10.1007/s10457-014-9701-x.

South, D.B. et J.L. Rakestraw. 2004. Large-diameter seedlings: A method of reducing chemical use in some pine plantations. J. Sustain. Forest. 18: 37-41. doi:10.1300/J091v18n04.

Takatsuki, S. 2009. Effects of sika deer on vegetation in Japn: A review. Biol. Coserv. 142(9): 1922-1929. doi:10.1016/j.biocon. 2009.02.011

Thiffault, N. 2004. Stock type in intensive silviculture: A (short) discussion about roots and size. For. Chron. 80(4): 463-468. doi:10.5558/tfc80463-4.

Thiffault, N. et V. Roy. 2011. Living without herbicides in Québec (Canada): Historical context, current strategy, research and challenges in forest vegetation management. Eur. J. For. Res. 130: 117133. doi:10.1007/s10342-010-0373-4.

Thiffault, N., B. Lafleur, V. Roy et J. DeBlois. 2012. Large planting stock type and mechanical release effects on the establishment suc- 
cess of Picea glauca plantations in Quebec, Canada. Intern. J. For. Res. 2012: 1-12. doi:10.1155/2012/617392.

Tremblay, J.-P., J. Huot et F. Potvin. 2006. Divergent nonlinear responses of the boreal forest field layer along an experimental gradient of deer densities. Oecologia 150(1): 78-88. doi:10.1007/ s00442-006-0504-2.

Tremblay, J.-P., J. Huot et F. Potvin. 2007. Density-related effects of deer browsing on the regeneration dynamics of boreal forests. J. Appl. Ecol. 44(3): 552-562. doi:10.1111/j.1365-2664.2007.01290.x.

Urli M., Barrette M., Leduc A. et Thiffault N. 2018. Résultats d'un délai d'application du dégagement mécanique en plantations d'épinettes blanche et noire dans un scénario de reboisement hâtif. For. Chron. 93(2): 183-194. doi: 10.5558/tfc2018-027.

Vourc'h, G., J.-L. Martin, P. Duncan, J. Escarré et T.P.C. Clausen. 2001. Defensive adaptations of Thuja plicata to ungulate browsing: a comparative study between mainland and island populations. Oecologia 126(1): 84-93. doi:10.1007/s004420000491.

Wagner, R.G., G.H. Mohammed et T.L. Noland. 1999. Critical period of interspecific competition for northern conifers associated with herbaceous vegetation. Can. J. For. Res. 29(7): 890-897. doi:10.1139/x99-055.
Ward, A.I. 2005. Expanding ranges of wild and feral deer in Great Britain. Mammal Rev. 35(2): 165-173. doi:10.1111/j.13652907.2005.00060.x

Ward, A.I., P.C.L. White, N.J. Walker et C.H. Critchley. 2008. Conifer leader browsing by roe deer in English upland forests: Effects of deer density and understorey vegetation. Forest Ecol. Manag. 256(6): 1333-1338. doi:10.1016/j.foreco.2008.06.034.

Ward, J.S., S.C. Williams et M.A. Linske. 2018. Influence of invasive shrubs and deer browsing on regeneration in temperate deciduous forests. Can. J. For. Res. 48(1): 58-67. doi:10.1139/cjfr-20170208.

Wardle, D.A., G.M. Barker, G.W. Yeats, K.I. Bonner et A. Ghani. 2001. Introduced browsing mammals in New Zealand naturel forests: Aboveground and belowground consequences. Ecol. Mono. 71(4): 587-614. doi:10.1890/0012-9615(2001)071[0587:IBMINZ] 2.0.CO;2. 